\title{
Role of nicergoline in corneal wound healing in diabetic rats
}

\author{
Amanda Lemos Barros Martins Portela1*, Rafael Neves Moreno', Maria Helena Madruga Lima Ribeiro², \\ Fernanda Miguel de Andrade', Yale Viana Alves ${ }^{3}$, Mônica Alves ${ }^{4}$ and Rodrigo Pessoa Cavalcanti Lira ${ }^{1}$
}

\begin{abstract}
Background: To investigate the effect of nicergoline on the rate of complete corneal ulcer reepithelialization (CCUR) in diabetic rats with diabetic keratopathy.

Methods: Forty-eight streptozotocin-induced diabetic rats were randomly divided into two groups. The experimental group $(n=24)$ received nicergoline $\left(10 \mathrm{mg} \cdot \mathrm{kg}^{-1}\right.$.day $\left.{ }^{-1}\right)$, while the control group $(n=24)$ received a placebo. A corneal epithelial defect was induced using a corneal diamond burr, and defect area was compared at time points of $0,12,24,48$ and $72 \mathrm{~h}$ after the injury using image analysis software. The probability of CCUR within $72 \mathrm{~h}$ was assessed using the Kaplan-Meier survival analysis log-rank test.

Results: When compared, 4 of the 24 rats (17\%) in the placebo group and 12 of the 24 rats (50\%) in the nicergoline group were found to have CCUR within $72 \mathrm{~h}$ (log-rank $=0.027$ ). Cox regression analysis found no effect of the covariates blood glucose $(P=0.601)$ or weight $(P=0.322)$ on the corneal reepithelialization (survival) curve.

Conclusions: Nicergoline increased wound healing rates relative to placebo and may therefore be investigated as a treatment option in diabetic keratopathy.
\end{abstract}

Keywords: Corneal epithelium, Diabetes mellitus, Diabetic neuropathy, Nicergoline, Wound healing, Corneal ulcer

\section{Background}

Diabetes affects 451 million people worldwide, approximately $70 \%$ of whom suffer from corneal complications known collectively as diabetic keratopathy. The most frequent clinical signs of diabetic keratopathy include punctate keratitis, recurrent epithelial erosions, and ulcers that are refractory to conventional treatments and which often fail to heal completely [1].

Previous controlled studies with corneal epithelial cell cultures in medium with high glucose levels have revealed that hyperglycemia over a period of $48 \mathrm{~h}$ is able to delay corneal reepithelialization to levels of only $29 \%$ of normal and to greatly increase the production of

\footnotetext{
* Correspondence: amandalbmportela@gmail.com

${ }^{1}$ Federal University of Pernambuco (UFPE), Recife, Pernambuco, Brazil

Full list of author information is available at the end of the article
}

reactive oxygen species [2]. The pathogenesis of diabetic keratopathy is multifactorial; prior studies have pointed to the impairment of the phosphatidylinositol 3-kinase (PI3K/AKT) transduction pathway, which responds to cell proliferation and migration in various systems and which also affects corneal healing [2]. In addition, oxidative stress and inflammatory cytokine production generated by hyperglycemia greatly contribute to the induction of cellular injury $[2,3]$.

In a previous study, rats with streptozotocin-induced diabetes exhibited a significant reduction in subepithelial basal nerve plexus density, as well as delayed closure of corneal epithelial ulcers 8 weeks after diabetes induction relative to controls [4].

Certain growth factors, cytokines, and neurotrophins are fundamental to corneal wound healing and act as regulators of cellular behavior and corneal epithelial

(c) The Author(s). 2021 Open Access This article is licensed under a Creative Commons Attribution 4.0 International License, which permits use, sharing, adaptation, distribution and reproduction in any medium or format, as long as you give appropriate credit to the original author(s) and the source, provide a link to the Creative Commons licence, and indicate if changes were made. The images or other third party material in this article are included in the article's Creative Commons licence, unless indicated otherwise in a credit line to the material. If material is not included in the article's Creative Commons licence and your intended use is not permitted by statutory regulation or exceeds the permitted use, you will need to obtain permission directly from the copyright holder. To view a copy of this licence, visit http://creativecommons.org/licenses/by/4.0/ The Creative Commons Public Domain Dedication waiver (http://creativecommons.org/publicdomain/zero/1.0/) applies to the data made available in this article, unless otherwise stated in a credit line to the data. 
regeneration. These include epidermal growth factor (EGF), fibroblast growth factor (FGF), substance P (SP), nerve growth factor (NGF), insulin-like growth factor-1 (IGF-1), and acetylcholine [5, 6]. New drugs targeting such factors have been investigated to treat diabetic keratopathy, since diabetes is associated with a significant decrease in the levels of these molecules [7-12].

Nicergoline (10a-methoxy-1,6-dimethylergoline-8 $\beta$ methanol-5- bromonicotinate, Sermion, Biogenesis AntiAging, Fish Hoek, South Africa) is an ergoline derivative indicated for cerebrovascular dementias with a broad mechanism of action. It acts as an $\alpha-1$ adrenergic antagonist, acetylcholinesterase inhibitor, dopaminergic agonist and PI3K/AKT pathway activator; it also increases SP and NGF levels and exhibits antioxidant properties. Such properties have inspired research into its use in corneal wound healing [13-18].

In a previous study, nicergoline contributed to the corneal reepithelialization of 110 eyes from 100 rats, and corneal NGF protein was higher in the nicergolinetreated group than in the control group [16]. In another experimental study that assessed 10 eyes from 5 healthy dogs without a control group, the effect of nicergoline on the ocular surface was assessed using corneal esthesiometry, Schirmer's test 1, and tear film break-up time, but the drug did not significantly alter canine ocular surface parameters [19].

In a prospective, noncomparative interventional study that included 27 eyes from 24 patients with neurotrophic keratopathy of multiple causes who had been unresponsive to conventional therapy, treatment with nicergoline contributed to the healing of neurotrophic corneal ulcers in $83 \%$ of eyes and improved Cochet-Bonnet corneal sensitivity and best-corrected visual acuity. In addition, tear NGF levels were significantly higher after nicergoline treatment [20].

Given the evidence that nicergoline may improve corneal healing processes in healthy animals, this study sought to evaluate the healing potential of nicergoline for the corneas of diabetic rats, and to assess its capacity to support the complete reepithelialization of corneal ulcers secondary to diabetic keratopathy.

\section{Methods}

\section{Ethical statements}

This study was approved by the Research Ethics Committee on Animal Research (CEUA) of the Federal University of Pernambuco (UFPE) and it was carried out in compliance with the ARRIVE guidelines. All procedures considering animals in this study adhered to the ARVO resolution for the care and use of animals in visual research.

\section{Experimental model}

The animals were acquired from the vivarium of the Department of Nutrition of the Federal University of Pernambuco and were housed in the vivarium of the KeizoAsami Immunopathology Laboratory (LIKA).

An experimental, controlled, and double-blind study design was employed with 48 male Wistar rats (Rattus norvegicus) between 8 and 10 weeks of age and weighing between 200 and $300 \mathrm{~g}$. They had no genetic modifications and had received all relevant vaccinations.

All animals had free access to appropriate feed and water and were housed in cages (4 rats per cage) with sawdust bedding under controlled temperature conditions $\left(24^{\circ} \mathrm{C} \pm 1{ }^{\circ} \mathrm{C}\right)$ and with a $12 \mathrm{~h}$ light-dark cycle (light switched on at 8:00 AM and switched off at 8:00 PM).

\section{Induction of diabetes mellitus}

The animals were housed in cages for 1 week before the start of the diabetes induction procedure. A single dose of $60 \mathrm{mg} \cdot \mathrm{kg}^{-1}$ of streptozotocin (Sigma-Aldrich, St. Louis, $\mathrm{MO}$ ) in a $0.5 \mathrm{M}$ sodium citrate buffer solution at a pH of 4.5 (Alfa Aesar Citrate, Fischer Scientific, Hampton, New Hampshire, USA) was administered through intraperitoneal injection [21].

In this experiment, $1 \mathrm{ml}$ syringes (26G) were used for the intraperitoneal injection of the streptozotocin buffer solution $12 \mathrm{~h}$ after food withdrawal (the animals continued to receive free access to water during this fasting period). As previous protocols have established, the induction of diabetes by streptozotocin triggers minimal pain and therefore does not require anesthesia [22].

Fifteen days after induction, each animal's weight and blood glucose levels were monitored periodically. Diabetes was confirmed by measuring blood glucose from a blood sample from the caudal vein with glucometer (Free Style Lite, Abbott, Chicago, Illinois, USA) $8 \mathrm{~h}$ after fasting. Diabetes was diagnosed if blood glucose was greater than or equal to $200 \mathrm{mg} \cdot \mathrm{dl}^{-1}$. Forty-eight diabetic animals were included in the study and randomly divided into the experimental nicergoline group $(n=24)$ and the placebo control group $(\mathrm{n}=24)$.

\section{Nicergoline treatment}

The 48 diabetic rats were treated by a blinded researcher 6 weeks after diabetes induction. The experimental group received nicergoline at a dosage of $10 \mathrm{mg} \cdot \mathrm{kg}^{-1}$. day $^{-1}$ that was diluted in each rat's drinking water. Meanwhile, the placebo group received drinking water without nicergoline. The animals' drinking water was routinely acidified using $\mathrm{HCl}$ to avoid the growth of pathogenic microorganisms; this acidification process is a sanitation measure typically used in vivariums and one which is safe for laboratory animals [16-18]. 
Oral administration was performed in both groups using gavage and manual containment 2 weeks prior to the corneal injury procedure, a protocol which was based on prior studies with a similar scope [16].

Radioisotope-marked nicergoline reaches peak serum radioactivity $3 \mathrm{~h}$ after oral administration. Its bioavailability is $5 \%$ of the total dose administered due to the first-pass effect, and it is predominantly eliminated in the urine. On average, $82 \%$ of marked nicergoline is eliminated in the urine and $10 \%$ in the feces as of $120 \mathrm{~h}$ after administration [19, 23].

Nicergoline was not administered in the 72 -h postoperative period; during this time, the animals were under periodic anesthesia/sedation for clinical evaluation and data collection.

\section{Corneal wound procedure}

Immediately after treatment (nicergoline or placebo), the animals were anesthetized through the intramuscularly administration of $10 \%$ ketamine hydrochloride (Cetamin, Syntec, São Paulo, Brazil) at a dose of $50 \mathrm{mg} . \mathrm{kg}^{-1}$ and $2 \%$ Xylazine Hydrochloride (Xilazin, Syntec, São Paulo, Brazil) at a dose of $10 \mathrm{mg} \cdot \mathrm{kg}^{-1}$; they also received a topical application of $0.4 \%$ oxybuprocaine hydrochloride eye drops (Oxinest, Cristalia Prod. Quim., Farm. Ltda, São Paulo, Brazil) in the left eye (OS). After anesthesia, superficial keratectomy surgery was performed on the left eyes by a blinded researcher.

The size of the corneal epithelial injury to be made was pre-established by using a corneal trephine $3.0 \mathrm{~mm}$ in diameter (Odous Instrumentos Ltda, Contagem, MG, Brazil). The area bound by the trephine was then deepithelialized with a corneal diamond burr (AlgerBrush II, Alger company, Lago Vista, TX), thus generating a central corneal defect, as described previously [4, 16, 24].

\section{Evaluation of corneal Reepithelialization}

To monitor corneal reepithelialization, the animals were anesthetized following the same procedure described previously and were evaluated at predetermined time points of $0,12,24,48$, and $72 \mathrm{~h}$ by a blinded researcher. The epithelial defect was evaluated by staining with $1 \%$ sodium fluorescein under cobalt blue filter and photographed using a Canon EOS Rebel T5i/EOS 700 D digital camera attached to the Opto SM Plus IBZ surgical microscope using an adapter. The area of the epithelial defect was measured by a different blinded researcher through photo analysis using Adobe Photoshop $[4,16,25]$.

Seventy-two hours after the procedure, the animals were euthanized using 3\% sodium pentobarbital (Hipnol, Syntec, São Paulo, Brazil) at an intramuscular dose of $150 \mathrm{mg} \cdot \mathrm{kg}^{-1}$ and monitored until heartbeats stopped.

\section{Statistical analysis}

Descriptive statistic data were calculated. The Kolmogorov-Smirnov normality test was used for continuous data. Means and standard deviations (SDs) were used for normally distributed data, and medians and interquartile ranges (IQR) were used for non-normally distributed data. Between-group differences in continuous variables were compared using the independent samples t-test for normally distributed data or using the Mann-Whitney U-test for non-normally distributed data.

The probability of reach complete corneal ulcer reepithelialization (CCUR) within $72 \mathrm{~h}$ was assessed using the Kaplan-Meier survival analysis log-rank test. Multivariate survival analysis was performed using the Cox regression model. The analyses were conducted using SPSS, version 21 (IBM Corporation, Armonk, NY, USA). $P$-values were two-tailed, and statistical significance was set at 0.05 .

\section{Results}

Demographic data are displayed in Table 1 and demonstrate homogeneity of the two groups in terms of weight and blood glucose.

The analyses revealed that 4 of the 24 rats $(17 \%)$ in the placebo group and 12 of the 24 rats $(50 \%)$ in the nicergoline group achieved complete reepithelialization of the cornea within $72 \mathrm{~h}$ (log-rank $=0.027)$. Cox regression analysis showed no influence of covariates on the survival curve of the corneal reepithelialization variable. The covariates assessed were glycemia $(P=0.601)$, weight $(P=0.322)$, and duration of diabetes $(P=0.208)$.

Table 2 details corneal ulcer reepithelialization progress. The area of the corneal ulcer at the 0 -h time point (immediately after surgery) was found to be consistent between the two groups: $6.42 \pm 1.17 \mathrm{~mm}^{2}$ in the placebo group and $6.07 \pm 1.31 \mathrm{~mm}^{2}$ in the nicergoline group $(P=$ 0.330 ).

Table 2 also shows that the area of the corneal ulcer was found to be significantly smaller in the nicergoline group at various time points. At the 12 -h time point, the area of the corneal ulcer in the nicergoline group $\left(4.71 \pm 1.53 \mathrm{~mm}^{2}\right)$ was smaller than that of the placebo group $\left(5.69 \pm 0.99 \mathrm{~mm}^{2} ; P=0.018\right)$.

The ulcers in the experimental group were also smaller at the 24-h time point: $3.15 \pm 1.65 \mathrm{~mm}^{2}$ in the nicergoline group compared to $4.52 \pm 1.56 \mathrm{~mm}^{2}$ in the placebo group $(P=0.012)$.

The findings were consistent at the 72-h time point as well, at which point the area of the corneal ulcer in the nicergoline group $\left(1.24 \pm 0.87 \mathrm{~mm}^{2}\right)$ was considerably lower than that of the placebo group $\left(3.51 \pm 3.48 \mathrm{~mm}^{2}\right.$; $P=0.017)$.

Figure 1 illustrates the corneal reepithelialization process in the two groups. Figure 2 shows an example of 
Table 1 Demographic data of the diabetic rats in the placebo and nicergoline groups

\begin{tabular}{llll}
\hline Clinical Value & Placebo $(\boldsymbol{n}=\mathbf{2 4})$ & Nicergoline $(\boldsymbol{n}=\mathbf{2 4})$ & $\boldsymbol{p}$-value \\
\hline Blood glucose, $^{\mathrm{a}}$ mean $(\mathrm{SD})$, median $\left(\mathrm{IQR}^{\mathrm{d}}\right), \mathrm{mg} / \mathrm{dL}$ & $351(81), 308(156)$ & $314(66), 309(95)$ & $0.085^{\mathrm{b}}$ \\
Weight, $^{\mathrm{a}}$ mean (SD), median (IQR), g & $230(39), 230(68)$ & $247(34), 250(53)$ & $0.103^{\mathrm{b}}$ \\
\hline
\end{tabular}

${ }^{a}$ normally distributed data; ${ }^{b}$ independent samples t-test, ${ }^{c}$ standard deviation, ${ }^{{ }}$interquartile range

$1 \%$ fluorescein staining of the corneal epithelial defect in the nicergoline and placebo groups immediately after surgery and at the 12-, 24-, 48-, and 72-h time points thereafter.

The median and interquartile range (IQR) of the reepithelialization rate in the first $72 \mathrm{~h}$ was $0.04(0.06)$ $\mathrm{mm}^{2} / \mathrm{h}$ in the placebo group and $0.08(0.03) \mathrm{mm}^{2} / \mathrm{h}$ in the nicergoline group $(P=0.055)$.

\section{Discussion}

The present study relied on an experimental model that reproduced insulin-dependent diabetes type 1 . The animals presented epithelial defects that failed to heal completely, thus resulting in the corneal ulcers commonly seen in cases of diabetic keratopathy.

Reepithelialization occurred twice as quickly in the experimental group as in the control group. Similar studies have found a 1.55 -fold increase in mean reepithelialization speed using a fibronectin-derived peptide eye drop treatment and a 1.47-fold increase using SP with insulinlike growth factor-1 eye drop treatment [24].

The two groups were also compared in terms of their ability to reach CCUR. Nicergoline was associated with significantly more corneal ulcer healing: after $72 \mathrm{~h}, 50 \%$ of the nicergoline group presented CCUR, while only $17 \%$ the of placebo group exhibited the same outcome (log-rank $=0.027$ ). Previous studies have also reported a delay in the reepithelialization of corneal defects in diabetic rats and a persistence of unhealed epithelial defects after $72 \mathrm{~h}$, and, in other studies, after more than $120 \mathrm{~h}[10,25]$.

The nicergoline group exhibited a statistically significant decrease in the area of the corneal ulcer at the 12$\mathrm{h}, 24-\mathrm{h}$, and 72-h time points. This finding suggests that nicergoline promotes corneal epithelial healing in diabetic rats and may be considered a promising new treatment option worthy of further investigation.

To date, this is the first experimental, controlled, and double-blind study to investigate the potential of nicergoline to improve the rate of corneal reepithelization and to treat corneal ulcers in a clinical context of diabetes, since the other studies in the literature evaluated this drug only in healthy animals $[13,14,20]$.

The current study exhibits some limitations. It is important to mention the lack of proof of diabetic neuropathy by an objective test, such as the corneal sensitivity test with the Cochet-Bonnet esthesiometer. We opted to forgo this procedure due to its low sensitivity and reproducibility [26, 27].

A second limitation was the type of intervention used (nicergoline vs. placebo) prior to corneal surgical injury. Nicergoline is a macromolecule that is currently only administered via the oral, intravenous, and intramuscular routes. It exhibits poor solubility in water $(0.002$ $\mathrm{mg} \cdot \mathrm{mL}^{-1}$ at $25^{\circ} \mathrm{C}$ ) in its commercial crystalline form; its hydrophobic and lipophilic properties therefore hinder its penetration into the hydrophilic corneal stroma, and its molecular size limits its ability to pass through the apical tight junctions of the epithelium. To resolve this problem, hydrolysis reactions may be used to modify the polarity of the drug; even so, the size of the molecule remains a limiting factor. Another solution is to change the size of nicergoline molecules through the use of nanoparticle assembly processes, which can reduce molecular size and allow for nanocrystals to be produced in an aqueous dispersion medium and stabilized using a surfactant or polymer [28].

Recent studies have reported on the use of nicergoline in the form of nanoparticles to be administered solely via the oral route; this process has been found to increase its solubility and, as a result, its bioavailability,

Table 2 Corneal reepithelialization in diabetic rats that underwent mechanical de-epithelialization after treatment with nicergoline or placebo

\begin{tabular}{|c|c|c|c|}
\hline \multirow[t]{2}{*}{ Measurement Time } & \multicolumn{2}{|c|}{ Epithelial Defect Area: mean (SDc), median (IQR) ${ }^{d}$ in $\mathrm{mm}^{2}$} & \multirow[t]{2}{*}{$p$-value } \\
\hline & Placebo $(n=24)$ & Nicergoline $(n=24)$ & \\
\hline$O h^{a}$ & $6.42(1.17), 6.20(1.28)$ & $6.07(1.31), 6.07(1.27)$ & $0.330^{\mathrm{e}}$ \\
\hline $12 \mathrm{~h}^{\mathrm{a}}$ & $5.69(0.99), 5.40(0.78)$ & $4.71(1.53), 4.68(2.51)$ & $0.018^{e}$ \\
\hline $24 \mathrm{~h}^{\mathrm{a}}$ & $4.52(1.56), 4.45(2.36)$ & $3.15(1.65), 3.30(3.08)$ & $0.012^{e}$ \\
\hline $48 \mathrm{~h}^{\mathrm{b}}$ & $3.57(3.21), 1.67(4.52)$ & $2.07(1.41), 0.50(2.00)$ & $0.095^{f}$ \\
\hline $72 h^{b}$ & $3.51(3.48), 0.97(4.38)$ & $1.24(0.87), 0.00(0.72)$ & $0.017^{f}$ \\
\hline
\end{tabular}

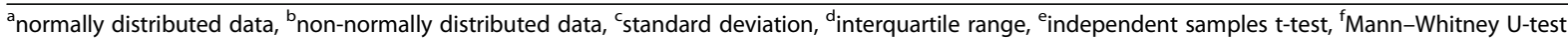




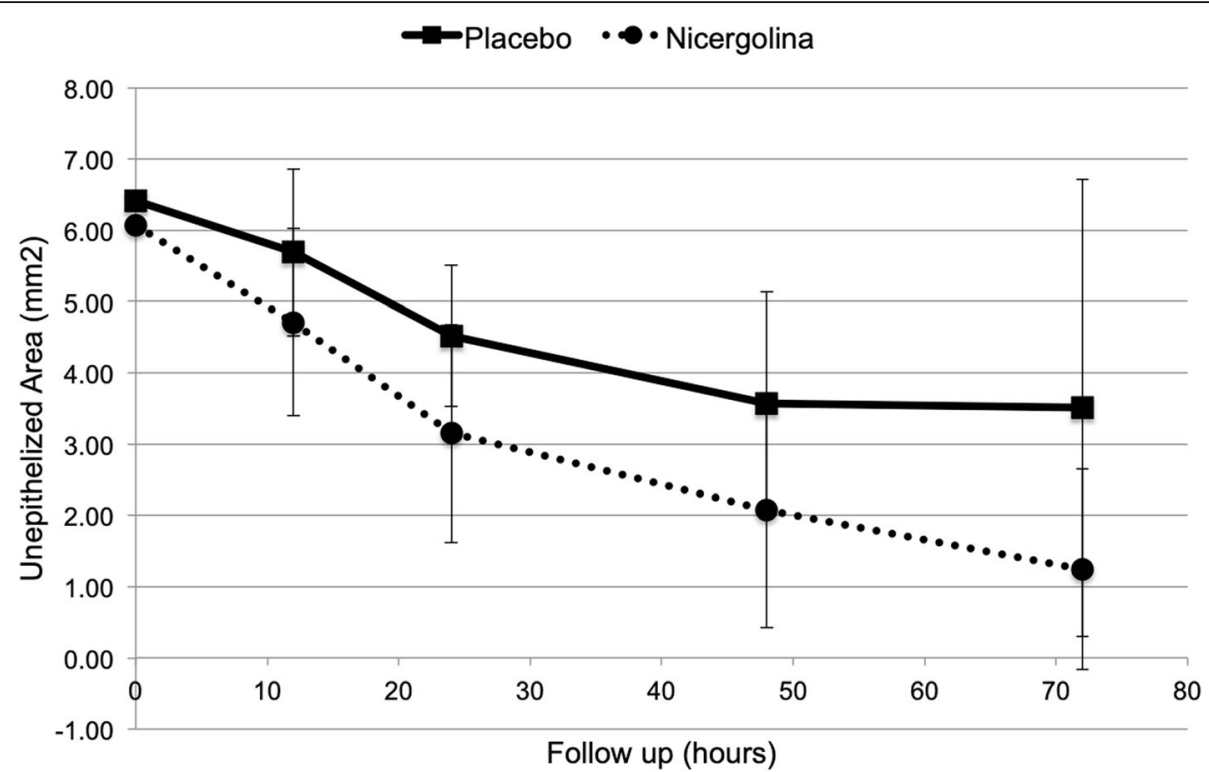

Fig. 1 Corneal reepithelialization progress in diabetic rats along the time points considered in eyes treated with nicergoline $(n=24)$ or placebo $(n=24)$

which is low (5\%) in the drug's commercial crystalline form. This type of pharmacological nanotechnology has been applied to certain anti-glaucoma drugs produced as eye drops, but it has not been applied to nicergoline as an eye drop. Thus, the use of nicergoline nanocrystals is currently limited to the oral route; a nicergoline eye drops were not available yet to be employed in studies. The findings in this study may inspire further research in the field of pharmacological technology in order to develop nicergoline-based nano eye drops [29, 30].

The animals' drinking water was suspended with nicergoline. To compensate for the drug's low solubility in water and consequently limited bioavailability, it was administered at a loading dose 2 weeks prior to the corneal injury procedure in order to prolong the animals' time of exposure to the drug. The nicergoline dosing regimen used in this study was similar to the methodology used in previous studies and was therefore considered appropriate $[16,29,30]$.

Another limitation was that post-treatment serum SP and NGF levels in the corneal tissue of the animals were not measured, but as described previously, an increase in SP and NGF in corneal tissue after nicergoline treatment has been previously observed in healthy animals $[16,17]$.

\section{Conclusions}

In conclusion, nicergoline was found to be associated with corneal wound healing in diabetic rats and likely contributed to the outcomes found herein. The rate of complete corneal ulcer reepithelialization after $72 \mathrm{~h}$ was higher among diabetic rats receiving nicergoline than in the placebo group. This evidence is promising, and more studies are needed to evaluate nicergoline as a potential treatment of corneal ulcers secondary to diabetic keratopathy.

Nicergoline
Fig. 2 Images of fluorescein corneal staining showing the area of epithelial defect in green along the time points considered in eyes treated with
nicergoline or placebo. The images are of an eye of a single animal from each study group




\section{Abbreviations}

CCUR: Complete corneal ulcer reepithelialization; PI3K AKT: Phosphatidylinositol 3-kinase AKT pathway; EGF: Epidermal growth factor; FGF: Fibroblast growth factor; SP: Substance P; NGF: Nerve growth factor; IGF-1: Insulin-like growth factor-1; CEUA: Research Ethics Committee on Animal Research; UFPE: Federal University of Pernambuco; CONCEA: National Council for the Control of Animal Experimentation; LIKA: Keizo-Asami Immunopathology Laboratory; OS: Left eye; SDs: Standard deviations; IQR: Interquartile range

\section{Acknowledgements}

The authors wish to thank the Keizo-Asami Immunopathology Laboratory (LIKA) and the Experimental Surgery Center of the Federal University of Pernambuco (UFPE) for their cooperation in conducting animal research.

\section{Authors' contributions}

R.N.M, M.H.M.L.R, F.M.A. and A.L.B.M.P contributed to induction of Diabetes Mellitus, nicergoline treatment, corneal wound procedure. Y.V.A. and A.L.B.M.P contributed to corneal reepithelialization analysis. R.P.C.L and A.L.B.M.P contributed to data analysis. M. A, R.P.C.L. and A.L.B.M.P. contributed to study design and manuscript preparation. The author(s) read and approved the final manuscript.

\section{Funding}

This research did not receive any specific grant from funding agencies in the public, commercial, or not-for-profit sectors.

\section{Availability of data and materials}

The datasets used and/or analysed during the current study are available from the corresponding author on reasonable request.

\section{Ethics approval and consent to participate}

This study was approved by the Research Ethics Committee on Animal Research (CEUA) of the Federal University of Pernambuco (UFPE) and registered under number 0021/2018. Animals underwent experimental procedures for scientific research purposes in accordance with the protocols published by the National Council for the Control of Animal Experimentation (CONCEA) and the ARRIVE guidelines. All procedures considering animals in this study adhered to the ARVO resolution for the care and use of animals in visual research.

\section{Consent for publication}

Not applicable.

\section{Competing interests}

The authors declare that they have no competing interests.

\section{Author details}

${ }^{1}$ Federal University of Pernambuco (UFPE), Recife, Pernambuco, Brazil. ${ }^{2}$ Keizo-Asami Immunopathology Laboratory (LIKA), Recife, Pernambuco, Brazil. ${ }^{3}$ Federal University of Campina Grande (UFCG), Campina Grande, Paraíba, Brazil. ${ }^{4}$ University of Campinas (UNICAMP), Campinas, São Paulo, Brazil.

Received: 11 November 2020 Accepted: 12 January 2021 Published online: 09 February 2021

\section{References}

1. Priyadarsini S, Whelchel A, Nicholas S, Sharif R, Riaz K, Karamichos D. Diabetic keratopathy: insights and challenges. Surv Ophthalmol. 2020;65(5):513-29.

2. Xu KP, Li Y, Ljubimov AV, Yu FS. High glucose suppresses epidermal growth factor receptor/phosphatidylinositol 3-kinase/Akt signaling pathway and attenuates corneal epithelial wound healing. Diabetes. 2009;58(5):1077-85.

3. Zhao $\mathrm{H}, \mathrm{He} \mathrm{Y}$, Ren $\mathrm{YR}$, Chen $\mathrm{BH}$. Corneal alteration and pathogenesis in diabetes mellitus. Int J Ophthalmol. 2019;12(12):1939-50.

4. He J, Pham TL, Kakazu A, Bazan HEP. Recovery of corneal sensitivity and increase in nerve density and wound healing in diabetic mice after PEDF plus DHA treatment. Diabetes. 2017:66(9):2511-20.

5. Di Zazzo A, Coassin M, Varacalli G, Galvagno E, De Vincentis A, Bonini S. Neurotrophic keratopathy: pros and cons of current treatments. Ocul Surf. 2019;17(4):619-23.
6. Maycock NJ, Marshall J. Genomics of corneal wound healing: a review of the literature. Acta Ophthalmol. 2014:92(3):e170-84.

7. Ljubimov AV, Saghizadeh M. Progress in corneal wound healing. Prog Retin Eye Res. 2015;49:17-45.

8. Park JH, Kang SS, Kim JY, Tchah H. Nerve growth factor attenuates apoptosis and inflammation in the diabetic cornea. Invest Ophthalmol Vis Sci. 2016;57(15):6767-75.

9. Ljubimov AV. Diabetic complications in the cornea. Vis Res. 2017;139:138-52.

10. Takamura Y, Matsumoto T, Tomomatsu T, Matsumura T, Takihara Y, Inatani M. Aldose reductase inhibitor counteracts the enhanced expression of matrix metalloproteinase-10 and improves corneal wound healing in galactose-fed rats. Mol Vis. 2013;19:2477-86.

11. Zagon IS, Sassani JW, Immonen JA, McLaughlin PJ. Ocular surface abnormalities related to type 2 diabetes are reversed by the opioid antagonist naltrexone. Clin Exp Ophthalmol. 2014;42(2):159-68.

12. Yang L, Di G, Qi X, et al. Substance P promotes diabetic corneal epithelial wound healing through molecular mechanisms mediated via the neurokinin-1 receptor. Diabetes. 2014;63(12):4262-74.

13. Zajdel P, Bednarski M, Sapa J, Nowak G. Ergotamine and nicergoline - facts and myths. Pharmacol Rep. 2015;67(2):360-3.

14. Zang G, Fang L, Chen L, Wang C. Ameliorative effect of nicergoline on cognitive function through the PI3K/AKT signaling pathway in mouse models of Alzheimer's disease. Mol Med Rep. 2018;17(5):7293-300.

15. Lee $\mathrm{S}, \mathrm{Na} \mathrm{SH}$, Chung YA, Jeong $\mathrm{H}$, Song IU. Efficacy of nicergoline treatment in Parkinson's disease associated with dementia. J Clin Neurosci. 2019;70:136-9.

16. Kim SY, Choi JS, Joo CK. Effects of nicergoline on corneal epithelial wound healing in rat eyes. Invest Ophthalmol Vis Sci. 2009;50(2):621-5.

17. Kim SY, Yang J, Lee YC. The effects of nicergoline on corneal nerve regeneration in rat corneas after photorefractive keratectomy. Curr Eye Res. 2011;36(1):29-33.

18. Kozlovac J, Schmitt B. Biosafety principles and practices for the veterinary diagnostic laboratory. Methods Mol Biol. 2015;1247:31-41.

19. Nishiyama Y, Abe A, Ueda M, Katsura K, Katayama Y. Nicergoline increases serum substance $P$ levels in patients with an ischaemic stroke. Cerebrovasc Dis. 2010;29(2):194-8.

20. Sebbag L, Moody LM, Allbaugh RA, Mochel JP. Nerve growth factor in dogs: assessment of two immunoassays and selected ocular parameters following a nicergoline challenge per os. Vet Ophthalmol. 2020;23(1):199-204.

21. Lee YC, Kim SY. Treatment of neurotrophic keratopathy with nicergoline. Cornea. 2015;34(3):303-7.

22. Furman BL. Streptozotocin-induced diabetic models in mice and rats. Curr Protoc Pharmacol. 2015:70:5.47.1-5.47.20.

23. Winblad B, Fioravanti M, Dolezal T, et al. Therapeutic use of nicergoline. Clin Drug Investig. 2008:28(9):533-52.

24. Morishige N, Uemura A, Morita Y, Nishida T. Promotion of corneal epithelial wound healing in diabetic rats by the Fibronectin-derived peptide PHSRN. Cornea. 2017;36(12):1544-8.

25. Moore K, Ghatnekar G, Gourdie RG, Potts JD. Impact of the controlled release of a connexin 43 peptide on corneal wound closure in an STZ model of type I diabetes. PLoS One. 2014;9(1):e86570.

26. Lum E, Murphy PJ. Effects of ambient humidity on the Cochet-bonnet aesthesiometer. Eye (Lond). 2018;32(10):1644-51.

27. Golebiowski B, Papas E, Stapleton F. Assessing the sensory function of the ocular surface: implications of use of a non-contact air jet aesthesiometer versus the Cochet-bonnet aesthesiometer. Exp Eye Res. 2011:92(5):408-13.

28. Ikuta Y, Aoyagi S, Tanaka Y, et al. Creation of nano eye-drops and effective drug delivery to the interior of the eye. Sci Rep. 2017:7:44229 Published 2017 Mar 14

29. Censi R, Gigliobianco MR, Casadidio C, Di Martino P. Changes in the solid state of Nicergoline, a poorly soluble drug, under different grinding and environmental conditions: effect on polymorphism and dissolution. J Pharm Sci. 2019:108(2):929-48.

30. Martena V, Shegokar R, Di Martino P, Müller RH. Effect of four different size reduction methods on the particle size, solubility enhancement and physical stability of nicergoline nanocrystals. Drug Dev Ind Pharm. 2014; 40(9):1199-205.

\section{Publisher's Note}

Springer Nature remains neutral with regard to jurisdictional claims in published maps and institutional affiliations. 